\title{
Application of the Principle of Maximum Entropy on rotor faults parameters.
}

\section{Gabriel Yuii Garoli (IC); Helio Fiori de Castro (PQ)}

\section{Abstract}

The modeling and analysis of rotor systems is important to understand and foresee its behavior. These rotor systems has stochastics characteristics, and then a stochastic analysis are needed. The maximum entropy principle is used to model the fault parameters and a Monte Carlo Method obtains the stochastic response.

Key words: rotordynamics, uncertainties, principle of maximum entropy

\section{Introduction}

Rotor system already presents uncertainties characteristic and the methods to identify and determinate its faults has uncertainties too. Therefore, a way to model these uncertainties and simulate is needed.

The principle of maximum entropy is applied to determinate the probability density function (pdf) of the rotor faults parameters, as residual unbalance mass and eccentricity of this unbalance mass. The faults considered were residual unbalance mass and bowed shaft. A Monte Carlo method was used to obtain the frequency response of the system and statistical information was determined.

\section{Results and Discussion}

The Principle of Maximum Entropy (PME) evaluate the pdf of a random variable, from the probabilistic momentums and the Shannon entropy is used as the non-information of the random variable. With that the pdf can be determined with the method of Lagrange multipliers.

$$
S(p)=\int_{k} p(x) \cdot \ln (p(x)) d x
$$

Equation 1. Shannon entropy

The support and the first two moments of the faults features was assumed and the pdf's was determined. Figure 1 is the pdf of the eccentricity of the unbalance mass, others pdf's was obtained for the others fault parameters.

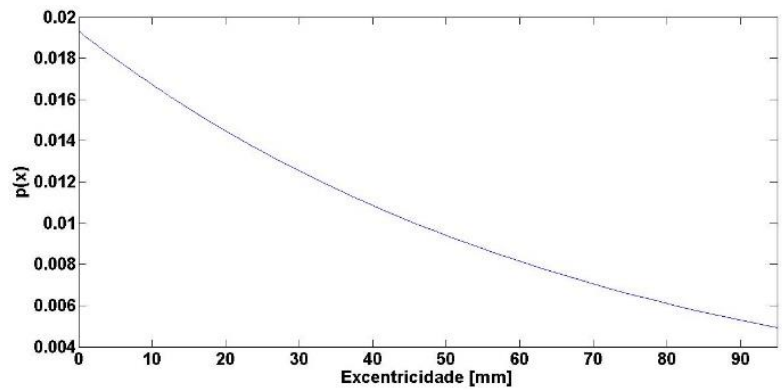

Figure 1. Pdf of the eccentricity of the unbalance mass.
Using the finite elements method and a Monte Carlo method, the stochastic response of a rotating system was determined and then, the mean and a confidence bound of $95 \%$ was calculated.

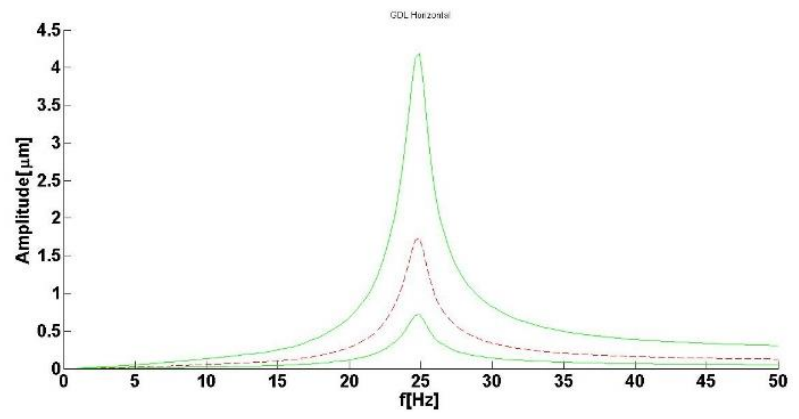

Figure 2. Pdf of the eccentricity of the unbalance mass.

\section{Conclusions}

The PME showed to be a good first approximation of the pdf of a random variable and could be used as the priori distribution of a Bayesian inference procedure.

The analyzed parameters of the faults affected considerably the amplitude of the response of the system. The effect on phase angle was considerable lower, comparing to the amplitude response.

\section{Acknowledgement}

The authors would like to thank FAPESP for support this research.

\footnotetext{
${ }^{1}$ Sampaio, R., Lima, R.Q., Modelagem Estocástica E Geração De Amostras De Variáveis E Vetores Aleatórios,SBMAC, 2012, vol 70
} 